Archives

\title{
L'image de la Tingitane historiographie arabe médiévale et antiquité maghrébine
}

\section{Ahmed Siraj}

\section{OpenEdition}

\section{Journals}

Édition électronique

URL : http://journals.openedition.org/ccrh/2698

DOI : $10.4000 /$ ccrh.2698

ISSN : 1760-7906

Éditeur

Centre de recherches historiques - EHESS

Édition imprimée

Date de publication : 4 octobre 1994

ISSN : 0990-9141

Référence électronique

Ahmed Siraj, «L'image de la Tingitane historiographie arabe médiévale et antiquité maghrébine », Les Cahiers du Centre de Recherches Historiques [En ligne], 13 | 1994, mis en ligne le 27 février 2009, consulté le 19 avril 2019. URL : http://journals.openedition.org/ccrh/2698; DOI : 10.4000/ccrh.2698

Ce document a été généré automatiquement le 19 avril 2019

Article L.111-1 du Code de la propriété intellectuelle. 


\title{
L'image de la Tingitane historiographie arabe médiévale et antiquité maghrébine
}

\author{
Ahmed Siraj
}

\section{NOTE DE L'AUTEUR}

Thèse dirigée par Jean Andreau (EHESS) et soutenue à l'Université de Paris I en janvier 1993 sous le titre de : L'Afrique du Nord antique à partir des textes arabes du Moyenagge. Histoire et géographie historique. Un exemple : le Maroc septentrional. Elle sera publiée prochainement dans la collection de l'École Française de Rome.

« Envisageons l'histoire dans sa forme extérieure: elle sert à retracer les évènements qui ont marqué le cours des siècles et des dynasties, et qui ont eu pour témoins les générations passées. Regardons ensuite les caractères intérieurs de la science historique : ce sont l'examen et la vérification des faits, l'investigation attentive des causes qui les ont produits, la connaissance profonde de la manière dont les évènements se sont passés et dont ils ont pris naissance... L'histoire a pour véritable objet de nous faire comprendre l'état social de l'homme, c'est-à-dire la civilisation... Or comme le mensonge s'introduit naturellement dans les récits historiques, ...la règle qu'il faut employer pour discerner dans les récits la vérité de l'erreur, règle fondée sur l'appréciation $d u$ possible et de l'impossible, consiste à examiner la société humaine c'est-à-dire la civilisation...En 
agissant ainsi, nous avons une règle sûre pour distinguer le vrai du faux, la vérité de l'erreur et cela par une méthode démonstrative qui ne laisse

aucune piste au doute. Nous avons ainsi un instrument qui permet d'apprécier les faits avec exactitude et qui pourra servir aux historiens qui dans leurs écrits tâchent de marcher dans la voie de la vérité... »

Ibn Khaldûn, Les Prolégomènes

1 A l'origine, cette recherche avait pour but de voir d'abord si, à partir des textes arabes, l'historien ou l'archéologue de la période classique peut trouver de nouvelles données permettent de compléter les lacunes de l'histoire et de la géographie historique de l'Afrique du Nord antique. Certes la nature et les caractères de la source arabe médiévale relative à la région ne sont pas comparables à celles des sources européennes du Moyenâge. Dans les milieux académiques, le mot-clé qui règne est celui de "rupture ». Sur plusieurs plans l'Afrique du Nord musulmane semble ne pas avoir gardé de liens avec celle que nous connaissons à travers les sources gréco-latines. Les transformations $\mathrm{du} \mathrm{VII}^{\mathrm{e}}$ siècle n'étaient pas un simple changement de patronage politique et militaire, mais une grande période d'interaction entre un Orient bédouin et un Maghreb profondément touché par les civilisations méditerranéennes. Cette rencontre était à l'origine d'une nouvelle orientation spirituelle et morale, si rapide et si efficace qu'elle a failli effacer toutes les traces des civilisations antérieures. Cette nouvelle vague orientale était-elle la continuation de l'antiquité, ou bien le point qui marque la rupture totale avec le passé ? L'opinion communément admise chez les chercheurs, notamment chez E. F. Gauthier, est celle qui reproche à la conquête arabe non pas seulement d'avoir été à l'origine d'une coupure dans l'histoire maghrébine, mais aussi d'avoir arrêté et désorienté l'évolution historique de la région. En fait, tout jugement sur la responsabilité de l'historiographie arabo-musulmane envers la période des ténèbres de l'histoire maghrébine reste arbitraire tant que des recherches sérieuses n'ont pas été entamées.

2 Notre recherche n'est, du point de vue des résultats pratiques, qu'un premier pas sur un chemin qui n'est certainement pas facile à suivre, celui qui essaye de rompre ce silence long et curieux relatif au passage de l'antiquité au haut Moyen-Age en Afrique du Nord. $\mathrm{Du}$ point de vue théorique, la démarche consiste à examiner la possibilité d'écrire l'histoire du passé à partir de beaucoup de sources historiques postérieures à la période que l'on veut étudier. Ce n'est pas une histoire au sens vrai du terme qui résulte d'une telle démarche. C'est plutôt un essai pour examiner de près l'image de l'histoire ancienne, nous entendons pré-islamique, chez les historiens musulmans. Autrement dit il s'agit d'une tentative visant à mettre en valeur les images de l'histoire c'est-à-dire la façon dont les différents peuples conçoivent et présentent l'histoire et les cultures des nations étrangères. L'expérience de l'historiographie arabe dans ce domaine est riche d'enseignements. L'une des nombreuses spécificités de cette historiographie, c'est celle qu'est censée présenter au lecteur une région qui tout en faisant partie de l'Umma, était très influencée par la civilisation latine. De quelles façons les historiens arabes allaient-ils présenter le passé de l'Afrique du Nord? Au-delà de ce qu'elle peut apporter comme information historique pure et simple, la réponse à cette question fournira un exemple des différentes conceptions que les nations du monde médiéval ont eues à l'égard des nations anciennes. 
Ce n'est donc plus l'histoire qui est l'objet de la recherche, c'est plutôt ce que nous avons appelé « l'historisation». Il n'y a pas que l'événement qui soit important, c'est tout le contexte qui entoure la façon dont on l'a conçu d'abord puis transmis ensuite qui doit tenir une place essentielle dans la réflexion historique.

4 Histoire, mémoire ou image de l'histoire ? Trois concepts différents que nous avons tenté au long d'une partie de notre étude d'extraire, de présenter et de commenter à partir d'un fond littéraire qui est, en fait, le miroir reflétant aussi bien le monde de l'Islam que la personnalité culturelle de ceux qui ont représenté son élite intellectuelle durant la période médiévale. Nous entendons par histoire le degré le plus simple de l'écriture historique à savoir la transmission des faits du passé tels qu'ils parviennent à l'historien à partir de ses sources. Par mémoire de l'histoire, la partie de l'histoire mémorisée, chez une nation quelconque, qu'elle soit relative à sa propre histoire ou bien à celle des nations étrangères. L'événement ou le fait historique mémorisé et reproduit est géré non pas seulement par le contexte objectif dans lequel il s'est produit dans le passé, mais aussi par celui dans lequel le "mémorisant l'évoque en tant que fait ou leçon historique "; L'image, elle, est la combinaison entre l'histoire ou ce qui en est mémorisé et l'imagination de l'historien traduite par l'interprétation qu'il propose pour tel ou tel fait.

Qu'il nous soit permis d'insister sur le fait que dès le moment où nous y sommes engagés, l'objectif n'a pas été de juger ou même d'évaluer une partie des informations historiques relatives à une région précise dans les sources arabes; c'était plutôt de recueillir ces informations pour compléter un vide dans l'histoire du Maghreb extrême. Au fur et à mesure de l'avancement de nos recherches, nous avons senti le besoin de classer ces informations et de tenter de découvrir le (ou les) discours qu'elles portent. Ces discours que nous avons tant bien que mal exposés, nous ont poussés à nous pencher sur le type de l'histoire que nous tentons de reconstruire à partir des textes arabes. Est-elle l'histoire telle qu'elle fut connue, telle qu'elle fut mémorisée ou telle qu'elle fut imaginée?

6 Evoquer l'histoire du Maghreb extrême par les sources arabes, c'est d'abord traduire une relation à un niveau purement intellectuel. D'où l'importance qu'il a fallu accorder à l'examen des rapports entre la terre étudiée et son historien. Rapports qui ne peuvent être conçus sans le retour aux origines du contact entre les deux mondes oriental et occidental. L'historien, au Moyen-Age, n'ayant été que le fruit d'un milieu très marqué par les exigences de sa propre culture aussi bien matérielle que spirituelle, fut peut-être l'homme le mieux placé pour servir de pont entre sa société et les nations du monde. Mais il a été aussi la victime de son métier. Car ce qu'il tente de présenter comme connaissances historiques n'est souvent pas ce que l'on appelle aujourd'hui « histoire», mais une interprétation, une image de celle-ci. Le Maghreb antique est peut-être l'un des exemples où cette méthode est bien illustrée.

7 Pour cette région, et ce n'est pas là un fait nouveau mais un phénomène qui est devenu «normal », la présence historique dont elle a bénéficié dans les écrits arabes médiévaux était née de l'action militaire du siècle de la conquête. C'est dire que cette histoire proposée a été élaborée après une longue période d'ignorance, et après une période « d'indifférence ». Le fruit d'une telle omission ne pouvait nous surprendre.

8 Au fur et à mesure que nous remontons dans le temps, les sources arabes deviennent de plus en plus profanes quant à la matière historique qu'elles fournissent. A commencer par la période du haut Moyen-Age très lacunaire, celle de la conquête très confuse, la période romaine et byzantine à peine connue, et les périodes précédentes complètement oubliées. 
Nous sommes donc loin d'écrire une histoire ancienne à partir des sources arabes. Ce ne sont que des débris qui reflètent, en fait, une conception ou une optique : celle qui est élaborée par l'historiographie arabe. Ce qui est important dans tout ce que nous avons tenté de reconstruire n'est donc pas «l'histoire de l'antiquité marocaine » avec ses faits et ses souvenirs médiocrement mémorisés par l'historien arabe, mais la façon dont elle est présentée ou plutôt représentée : c'est son image.

Cette histoire se compose de deux parties : l'histoire mémorisée et l'histoire imaginée. Le rapport entre ces deux catégories est un rapport de causalité : la marge de l'ignorance des faits historiques de l'Antiquité a servi au développement d'une histoire purement mythologique qui a caractérisé l'image du Maghreb extrême chez l'historien arabe, et bien évidemment dans son écrit. Certes, comme chez les auteurs de l'Antiquité, le facteur géographique a joué un rôle important. C'est à cause de lui que ce Maghreb extrême, situé aux confins de l'inconnu est devenu terre de légendes où les héros de la mythologie venaient tous camper.

Si l'on considère l'ensemble des données recueillies dans les sources arabes, nous nous rendons compte facilement de l'importance de leur contribution à la connaissance de l'histoire et de la géographie historique du Maroc préislamique. L'un des obstacles de l'historiographie arabo-musulmane était justement cette absence d'une vision historique qui va au-delà de la simple présentation des faits de l'Antiquité à titre de leçons. Certes l'historien médiéval, en raison de son milieu socio-culturel, n'a pas pu se libérer du sentiment religieux. ce dernier a toujours constitué la structure fondamentale qui encadre sa pensée historique. Dans les passages consacrés à l'histoire ancienne du Maghreb persistent les traces profondes du discours religieux dans les récits des historiens arabes. Cela ne signifie pas pour autant qu'il y ait eu une négligence de l'histoire ancienne en tant que discipline faisant partie de l'adab et plus précisément en tant que spécialité liée généralement à l'histoire universelle. Les plus grands historiens ont tous réservé des chapitres à l'histoire des nations anciennes. Cette méthode est devenue une tradition dans leurs écrits. Si le Maghreb n'est pas considéré dans cette discipline, c'est parce qu'il n'a jamais constitué une grande nation qui ait contribué d'une manière remarquable aux évènements de l'Antiquité au même titre que la Chine, l'Inde, Rome ou Byzance. Son histoire n'apparaissait souvent qu'à travers celles des peuples qui l'ont dominé. Pour des historiens comme al-Mas ûdî, al-Tabarî ou Ibn al-Athîr, dont l'intérêt s'est orienté souvent vers la recherche des grandes civilisations du passé, auxquelles la civilisation arabe venait se substituer, il n'était pas important de prendre en considération dans leur inventaire des nations du mode la nation berbère dont l'histoire n'a pas eu, à partir de l'espace géographique connu sous le nom du Maghreb, une dimension ou une tendance universelle.

11 Cet état de fait fut peut-être à l'origine de l'éveil de l'esprit khaldounien qui, tout en écrivant une histoire universelle, tenait le plus grand compte de celle des Berbères. Et c'est chez cet historien qu'on trouve les informations les plus «riches» sur le monde berbère préislamique. Cependant, nous avons vu à travers l'étude consacrée à ces informations qu'il s'agissait en fait d'une «image » très ambiguë, bien qu'elle fût très représentative d'une attitude culturelle issue de la période de la conquête. C'est le même phénomène qu'on relève dans toutes les productions culturelles étrangères concernant le Maroc, à commencer peut-être même par le Périple d'Hannon et jusqu'à l'historiographie française de la période coloniale, en passant évidemment par les sources gréco-latines. Ce 
n'est qu'une suite d'images à travers lesquelles l'historien d'aujourd'hui tente, tant bien que mal, d'écrire « une histoire », pour ne pas dire « des histoires ».

S'il est impossible d'écrire une histoire de l'Afrique du Nord antique proprement dite à partir des textes arabes médiévaux, la contribution de ces derniers à la connaissance de la géographie historique du Maroc antique est d'une grande valeur.

Sur le terrain, une autre réalité existait : les vestiges importants d'une civilisation passée. L'historien n'a cependant pas été confronté à eux, puisqu'il est en principe un intellectuel dont le métier historiographique est fondé sur les connaissances acquises à partir de la lecture et de la tradition orale. Le terrain appartient au géographe, homme de voyage, qui, en plus des deux sources utilisées par l'historien, était amené lors de ses différentes pérégrinations à découvrir quotidiennement les régions parcourues. Toutefois, pour l'histoire qui nous intéresse, il est un mauvais connaisseur. Remarquons qu'al-Bakrî qui fournit une liste des sites antiques de l'Afrique du Nord et dont la plupart sont des sites romains, n'évoque presque jamais les Romains et ne cherche généralement jamais à identifier les bâtisseurs de ces sites, mêmes s'ils sont remarquables. La même remarque est valable pour la majorité des géographes du Moyen-Age proprement dit. Dans ce groupe d'écrivains, Jean Léon l'Africain semble plutôt représenter un cas particulier. Ayant été à Rome, et ayant élaboré ses connaissances sur l'histoire de l'Afrique antique, il tente d'une façon presque systématique d'identifier les sites décrits. Une conclusion reste évidente quant à la conception des ruines antiques chez les géographes voyageurs : pour ces derniers, un site antique n'est pas un indice historique mais un repère topographique.

Malgré son importance capitale et sa contribution à la connaissance de l'évolution historique de la région, la géographie historique du Maroc médiéval est un terrain encore vierge. Les descriptions arabes sont les seules sources littéraires succédant aux sources classiques et concernant une période qui n'avait pas encore coupé tous les liens avec l'Antiquité. Elles seraient donc capables de fournir des données relatives à la géographie historique de la région. D'où l'importance que nous avons accordé à cette partie de l'étude. Nous croyons qu'à présent l'étude doit s'orienter de plus en plus vers le terrain.

15 L'image géographique du Maroc du nord du Moyen-Age est, évidemment, une version évoluée de la carte antique. Certes, plusieurs changements peuvent être relevés, mais nous avons vu que certains aspects de la géographie classique ont subsisté pendant la période arabe. Ainsi, le problème des rapports terrestres entre le Maroc et le reste de l'Afrique du Nord à travers le couloir de Taza reste un sujet auquel il faut penser dans le futur. Au haut Moyen-Age, nous l'avons démontré à travers l'étude de l'itinéraire d'al-Ya kûbî, les Arabes ont empruntés les chemins du Rif. Rien n'empêche de croire que les raisons de cette absence d'un itinéraire à travers le couloir de Taza doivent être cherchées dans les évènements de la période romaine. L'étude des itinéraires a révélé l'importance des sources arabes pour la connaissance de la géographie antique. Il ne faut pas oublier que l'espace géographique auquel nous nous sommes intéressés (le Maroc du nord) représente la partie de l'Afrique romaine où les itinéraires antiques sont encore mal connus. La carte des itinéraires médiévaux telle qu'elle fut établie à partir des données des géographes occidentaux, notamment al-Bakrî, pourrait constituer une base pour les prochains travaux de prospection.

16 L'un des mérites de cette recherche, c'est de mettre en évidence, par delà le fossé des siècles obscurs qui d'ailleurs commence bien avant l'Islam pour la région, la continuité de plusieurs problèmes à la fois géographiques et historiques et d'un certain nombre 
d'attitudes intellectuelles ou de comportements pratiques à leur propos depuis l'Antiquité jusqu'au Moyen-Age.

17 A partir d'une étude qui met à contribution à la fois les sources arabes et les sources latines, on arrive à combler certaines lacunes dont l'importance est parfois capitale pour l'histoire de la Mauritanie occidentale romaine et post-romaine. Signalons parmi les résultats de notre recherche l'identification de la tribu des Awraba avec les Orbicae de Ptolémée, ce qui a permis de mettre fin aux débats sur sa localisation et sur son rôle politique au Maroc du nord depuis le retrait de l'administration romaine vers la fin $\mathrm{du}_{\mathrm{III}} \mathrm{e}^{\mathrm{e}}$ s. jusqu'à l'arrivée des Idrissides.

18 L'apport des textes arabes à l'étude de l'évolution des cités romaines, à leur localisation et à leur identification a été démontré d'une façon incontestable. L'idée largement répandue parmi les historiens archéologues selon laquelle les Musulmans avaient l'habitude de construire leurs villes loin des sites antiques pour éviter toute confusion entre leur civilisation islamique et celle de Rome, est plus que jamais mise en cause. Car à partir des informations des géographes arabes, on peut établir une chronologie du site de Volubilis qui semble avoir continué à jouer son rôle de chef-lieu du Maroc du Nord après le viI ${ }^{\mathrm{e}}$ siècle et jusqu'au XII ${ }^{\mathrm{e}}$ siècle. La même remarque est valable pour Sala et surtout pour Tingis, dont les textes arabes confirment sans aucun doute possible qu'il avait le statut de capitale politique de la Tingitane.

De même, ces textes géographiques nous ont permis parfois de localiser quelques sites inédits. C'est l'exemple du site antique signalé par al-Bakri dans la région d'Ouezzane (région où l'occupation romaine n'est jamais évoquée) et que nous avons proposé d'identifier à la colonie romaine Babba Inliz Compestris.

Histoire et géographie historiques sont les deux axes qui ont guidé notre recherche. Le but était bien évidemment de mettre au jour l'image du Maroc antique telle qu'elle est représentée dans l'historiographie arabe. A travers cette image, serait-il possible de trouver des rapports entre les deux périodes, romaine et arabe en Afrique du Nord? Autrement dit : Y-a-t-il une rupture ou une continuité dans l'histoire de la région?

L'idée d'une rupture dans l'histoire de la région a longtemps dominé l'esprit des chercheurs. En fait, tant par préjugés idéologiques que par rigidité de la spécialisation scientifique, l'Afrique du Nord romaine et le Maghreb arabe paraissent aux yeux des historiens comme étant deux mondes complètement différents, où le fil de la continuité s'interrompt au moment même où la région change de maître. Nous croyons que la présente thèse a apporté des éléments qui attestent sans aucun doute possible la continuité de l'histoire et de la géographie historique de la région. La rupture existe certainement ; non pas dans l'histoire de la région, mais dans les sources de cette histoire. Nous entendons par là celles qui concernent l'intervalle chronique séparant la fin de la période romaine en Tingitane du début de la période arabe.

Cette étude avait pour objectif primordial de voir, à partir d'une lecture exhaustive et attentive des textes en question, quelles étaient les connaissances des Arabes à propos des antiquités africaines, et quelles étaient les diverses interprétations et les "jugements » portés par ces auteurs. Dès lors, nous avons réalisé l'importance capitale d'une étude sur l'attitude intellectuelle de ces auteurs envers les anciennes civilisations et cultures méditerranéennes et orientales. Comment ont-ils conçu et transmis ces connaissances relatives à l'Antiquité ? Certes, il s'agit tout d'abord d'un travail d'historiographie qui met à contribution plusieurs disciplines. Histoire politique, histoire religieuse, histoire 
économique, histoire de l'évolution des mentalités : bref, il faut d'abord commencer par comprendre le texte et son auteur avant d'envisager l'étude du discours ou des discours, de l'écrit. C'est dire qu'il faut faire tout un travail d'historiographie visant à déterminer non pas seulement les dimensions traditionnelles du texte mais aussi, et surtout, de déterminer les circonstances, les motivations et les objectifs de sa mise à jour. D'abord, il faut certainement pousser plus loin les recherches relatives à la naissance de l'historiographie arabe. Il ne s'agit pas de connaître quand ni comment les premiers écrits historiographiques furent réalisés, mais pourquoi les Arabes ont décidé à un moment précis de leur histoire d'écrire l'histoire des Autres ? Quelle histoire ont-ils écrite ? Quelle limite géographique, culturelle et religieuse ont-ils respectée? A quels discours se sont-ils attachés ? Bref quel sens donnaient-ils à cette discipline?

Ce n'est qu'après avoir réalisé cet essai d'interprétation sur la naissance de l'historiographie arabe qu'il serait possible de comprendre l'évolution. Le fait qu'un historien comme al-Mascûdî accorde beaucoup plus d'importance à la civilisation grecque qu'à celle de Rome n'est certainement pas le résultat d'un pur hasard, mais plutôt d'une réalité qui trouve son explication d'une part dans l'évolution politique du monde musulman, et d'autre part dans l'évolution (ou la révolution) culturelle due au contact avec les civilisations étrangères. Il faut revenir aux origines de la production historiographique arabe. Entreprise difficile puisque la plupart des oeuvres qui marquent la naissance de l'histoire chez les Arabes sont perdues. Dans les meilleurs cas, quelques passages subsistent chez des disciples ou des historiens postérieurs. Il est évident qu'une enquête sur les thèmes abordés par les premiers historiens a sa valeur. Car, il faut dans tous les cas arriver à déterminer la date approximative de l'apparition des peuples étrangers dans les écrits arabes.

Une fois que ce tableau sera dressé, il va falloir répondre à l'inévitable question: comment a-t-on écrit cette histoire? A partir de quelles sources? Suivant quelle méthode ? Il est établi qu'aux VIII ${ }^{e}$ et $\mathrm{x}^{\mathrm{e}}$ siècles des intellectuels arabes ont pu consulter des sources grecques, latines, perses... qui circulaient dans l'empire de l'Islam. Il est possible, voire même probable, que ce contact culturel a commencé plus tôt au fur et à mesure du progrès de la conquête notamment aux confins du territoire de l'Islam. De toute façon, il est certain que les historiens arabes, surtout les tout premiers, n'étaient pas enthousiastes pour la connaissance des peuples étrangers, les Méditerranéens en particulier. L'une des urgences pour ces intellectuels était de connaitre leur propre histoire, de la rédiger, de la glorifier... et si cette histoire écrite n'existe pas, il aurait fallu la créer, l'inventer, l'élaborer pour que la nation arabe soit digne d'affronter, sur le plan culturel, les grandes nations du monde.

Dans ce grand mouvement $d u$ VIII ${ }^{\mathrm{e}}-\mathrm{IX} \mathrm{e}^{\mathrm{e}}$ siècle (nous insistons sur cette période, car c'est d'ici que les «choses » commencent) quelques noms se distinguent. Qui ne connaît pas Tabarî, Ibn Abd al-Hakam ou al-Mascûdi ? Ce sont des vrais représentants de l'aube de l'historiographie arabe. Leur intérêt réside dans le fait que chacun d'eux représente un point sur la ligne d'une évolution; chacun d'eux représente une école. Mais il ne faut que ces grands noms qui, pour plusieurs raisons, dominent les études relatives à l'historiographie arabe cachent d'autres noms, moins connus certes, mais qui peuvent être très utiles sur certains points qui concernent notre thème. Il faut donc procéder à une " prospection " générale de la production d'historiographie arabe.

Après la conquête, le noyau arabe (nous entendons par là le centre primitif de l'empire de l'Islam) se trouvait entouré d'autres peuples ayant des cultures très développées et dont 
certains sont des sujets de Médine, de Damas ou de Bagdad; Les premiers contacts avec ces civilisations ont obéi à la force des armes. Le phénomène de la conquête ne pouvait se dérouler sans laisser des traces marquantes chez l'intellectuel. C'est dire que cette conquête a influencé d'une façon ou d'une autre l'attitude de l'historien envers les civilisations étrangères. Cette attitude s'est traduite par une image qui a été élaborée durant des siècles par les historiens. Comme dans toute société de colonisation, les rapports entre les dominants et les dominés se caractérisent par une sorte de mépris de la part des premiers envers les seconds. Chez les Arabes cette attitude s'appuie sur un sentiment de supériorité notamment dans le domaine religieux. Les Arabes sont ceux qui ont engendré l'ultime messager de Dieu, celui qui a révélé la vérité divine la plus valable qui abolit tout ce qui précède. C'est cette vérité, l'Islam, qu'il faut transmettre aux peuples du monde et qu'il faut imposer s'il le faut par la force des armes.

Dans ces travaux des premiers temps de l'Islam, le lecteur trouve une présentation des mondes anciens et contemporains (par rapport à l'époque), avec les principaux faits et les événements qui marquent l'évolution. Ces rapports avaient d'abord pour objectif de faire connaître le nouveau monde à la nation arabe, donc c'est un acquis de conscience; ensuite, il n'était pas question uniquement de présenter les autres à l'honnête homme arabe, mais en plus d'interpréter leur histoire, puisqu'il s'agit au fond d'une composante essentielle de leur identité, et donc un moyen ou un obstacle de leur intégration dans le nouveau monde. A partir de là, la tentative des auteurs arabes devient intéressante, car l'on voit créer des images multiples de l'Autre, qu'il soit ou non membre de la communauté : la Umma. Comment a-t-on procédé à l'élaboration de ces essais? Comment se présentent-ils? A-t-on réussi le discours, Comment s'est-on présenté aux autres et comment a-t-on présenté ces autres ? Ce sont toutes des questions qui permettent de voir de près un stade important dans l'évolution des contacts méditerranéens.

Notre recherche a pour objectif à la fois d'établir un nouveau rapport entre le texte classique en tant que source de l'histoire et de l'historien en tant qu'enquêteur qui cherche à comprendre ses propres outils de travail avant de comprendre l'histoire recherchée, et d'autre part d'ouvrir de nouveaux horizons pour l'exploitation de ces sources. Le projet se compose de deux parties.

1. L'archéologie des sources. Outre la présentation du dossier de sources, il est évident qu'il va falloir s'attacher aux problématiques que nous avons déjà abordées ci-dessus. Il faut insister notamment sur les problèmes de la méthode suivant laquelle il faut manipuler ces sources, comment il faut les classer, et surtout comment il faut classer leurs informations (ce que nous appelons « l'archéologie du texte »).

2. L'image des civilisations antiques dans les sources arabes du Moyen-Age. Il est impossible de dresser un tableau général sur l'ensemble des peuples qui ont fait l'objet d'une présentation par l'historien arabe. Nous limiterons l'étude dans un premier temps à deux régions qui représentent deux civilisations différentes: le Maghreb des Berbères qui présente le cas d'une civilisation non arabe mais intégrée au monde de l'Islam; et la civilisation gréco-romaine qui présente le cas d'une civilisation restée en dehors des limites du monde de l'Islam. 